\title{
Analysis of genes and underlying mechanisms involved in foam cells formation and atherosclerosis development
}

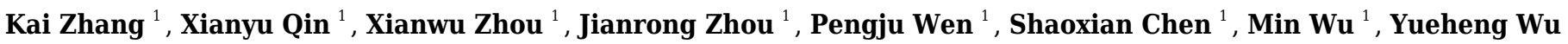 \\ Corresp., 1 , Jian Zhuang ${ }^{\text {Corresp. } 1}$ \\ ${ }^{1}$ Department of Cardiovascular Surgery, Guangdong Provincial Key Laboratory of South China Structural Heart Disease, Guangdong Provincial People's \\ Hospital \& Guangdong Academy of Medical Sciences, School of Medicine, South China University of Technology, Guangdong Cardiovascular Institute, \\ Guangzhou, Guangdong, China \\ Corresponding Authors: Yueheng Wu, Jian Zhuang \\ Email address: edgar_wuyh@outlook.com, zhuangjiangenetics@163.com
}

Background: Foam cells (FCs) play crucial roles in the process of all stages of atherosclerosis. Smooth muscle cells (SMCs) and macrophages are the major sources of FCs. This study aimed to identify the common molecular mechanism in these two types of FCs. Methods: GSE28829, GSE43292, GSE68021, and GSE54666 were included to identify the differentially expressed genes (DEGs) associated with FCs derived from SMCs and macrophages. Gene Ontology biological process (GO-BP) and Kyoto Encyclopedia of Genes and Genomes (KEGG) pathway analyses were performed by using the DAVID database. The co-regulated genes associated with the two origins of FCs were validated (GSE9874), and their expression in vulnerable atherosclerosis plaques (GSE120521 and GSE41571) was assessed. Results: A total of 432 genes associated with FCs derived from SMCs (SMCFCs) and 81 genes associated with FCs derived from macrophages (M-FCs) were identified, and they were mainly involved in lipid metabolism, inflammation, cell cycle/apoptosis. Furthermore, three co-regulated genes associated with FCs were identified: GLRX, RNF13, and $A B C A 1$. These three common genes showed an increased tendency in unstable or ruptured plaques, although in some cases, no statistically significant difference was found. Conclusions: DEGs related to FCs derived from SMCs and macrophages have contributed to the understanding of the molecular mechanism underlying the formation of FCs and atherosclerosis. GLRX, RNF13, and ABCA1 might be potential targets for atherosclerosis treatment. 


\section{Analysis of genes and underlying mechanisms involved in foam cells formation and atherosclerosis development}

Kai Zhang, Xianyu Qin, Xianwu Zhou, Jianrong Zhou, Pengju Wen, Shaoxian Chen, Min Wu,

6 Yueheng $\mathrm{Wu}^{*}$, Jian Zhuang*

Department of Cardiovascular Surgery, Guangdong Cardiovascular Institute, Guangdong

9 Provincial Key Laboratory of South China Structural Heart Disease, Guangdong Provincial

People's Hospital \& Guangdong Academy of Medical Sciences, School of Medicine, South

11 China University of Technology, Guangzhou, China

*Correspondence to:

Jian Zhuang \& Yueheng Wu.

Department of Cardiovascular Surgery, Guangdong Cardiovascular Institute, Guangdong

Provincial Key Laboratory of South China Structural Heart Disease, Guangdong Provincial

People's Hospital \& Guangdong Academy of Medical Sciences, School of Medicine, South

\section{Abstract}


Background: Foam cells (FCs) play crucial roles in the process of all stages of atherosclerosis.

Smooth muscle cells (SMCs) and macrophages are the major sources of FCs. This study aimed

to identify the common molecular mechanism in these two types of FCs.

Methods: GSE28829, GSE43292, GSE68021, and GSE54666 were included to identify the differentially expressed genes (DEGs) associated with FCs derived from SMCs and macrophages. Gene Ontology biological process (GO-BP) and Kyoto Encyclopedia of Genes and Genomes (KEGG) pathway analyses were performed by using the DAVID database. The coregulated genes associated with the two origins of FCs were validated (GSE9874), and their expression in vulnerable atherosclerosis plaques (GSE120521 and GSE41571) was assessed.

Results: A total of 432 genes associated with FCs derived from SMCs (SMC-FCs) and 81 genes associated with FCs derived from macrophages (M-FCs) were identified, and they were mainly involved in lipid metabolism, inflammation, cell cycle/apoptosis. Furthermore, three co-regulated genes associated with FCs were identified: $G L R X, R N F 13$, and $A B C A 1$. These three common genes showed an increased tendency in unstable or ruptured plaques, although in some cases, no statistically significant difference was found. $G L R X, R N F 13$, and $A B C A 1$ might be potential targets for atherosclerosis treatment.

Keywords: Foam cells, Atherosclerosis, Smooth muscle cells, Macrophages, Differentially expressed genes 
61 Introduction

62 Atherosclerosis is a complex chronic disease characterized by the thickening of the intima

63 and the formation of atherosclerotic plaques, leading to asymmetric stenosis of the arterial 
64 lumen(Wang et al. 2019a). It is the underlying pathophysiological mechanism of many cardiac-

65 cerebrovascular diseases, including coronary arterial disease, ischemic stroke, and other

66 peripheral arterial diseases, which are the leading causes of death in the world(Katakami 2018;

67 Wang et al. 2019a). The pathogenesis and molecular mechanisms involved in atherosclerosis are

68 multifactorial, including endothelial dysfunction, abnormal smooth muscle cell (SMC)

69 proliferation and migration, oxidized lipid deposition, vascular matrix changes, inflammatory

70 cell infiltration, and oxidative stress(Weber \& Noels 2011; Zhou et al. 2019). In recent decades,

71 significant improvements have been made in the diagnosis, prevention, and treatment of

72 atherosclerosis. However, the comprehensive and in-depth molecular mechanisms of

73 atherosclerosis still need to be further explored(Kanter et al. 2018).

74 Intra- and extracellular lipid deposition is a crucial trigger for the development of

75 atherosclerotic lesions(Summerhill et al. 2019), while lipid-rich foam cells (FCs) are the earliest

76 manifestations of atherosclerotic lesions, and their emergence and accumulation are the original

77 causes of atherosclerotic plaques(Olivares et al. 2016). It was previously thought that all FCs in

78 human atherosclerotic lesions were derived from macrophages. However, subsequent studies of

79 human arterial plaques showed that cell types inherent to the arterial wall are also involved in the

80 formation of FCs, including SMCs, stem/progenitor cells, and even endothelial cells(Gisterå \&

81 Hansson 2017; Maguire et al. 2019). SMCs and macrophages are the two main sources of FCs in

82 arterial plaques. FCs formation in atherosclerosis development is closely related to imbalances in

83 lipid metabolism, including lipid uptake and/or lipid efflux inhibition(Maguire et al. 2019;

84 Poznyak et al. 2020). 
The purpose of "omics" research is to integrate and analyze a large amount of information that represents the overall biological parameters in a particular state through advanced computer technology to identify some important information related to phenotypes and/or diseases(Vernon et al. 2019). A single biomarker may not be sufficient to represent the complex biological processes of the disease, and omics methods can be used to capture multiple variables and demonstrate the interrelationship between those variables in the disease process(Vernon et al. 2019). In recent years, the expression profiles of atherosclerosis have been determined by omics approaches, such as microarrays and RNA-seq, and hundreds of differentially expressed genes (DEGs) involved in the development of atherosclerosis have been identified(Liu et al. 2020; Tan et al. 2017). Recently, many studies have attempted to explore the possible molecular mechanisms of macrophage-derived FCs (M-FCs) through microarray technology and bioinformatics analysis(Hägg et al. 2008; Huang et al. 2019; Reschen et al. 2015). However, few studies have systematically explored the molecular mechanism of foam cells derived from smooth muscle cells (SMC-FCs) through high-throughput methods. In this study, bioinformatics techniques were utilized to investigate the possible mechanisms of SMC- and macrophagederived FCs to identify the common molecular mechanisms of these two different sources of FCs.

\section{Methods and materials}

\section{Data resources}


106

107

108

109

110

111

112

113

114

115

the National Center for Bioinformatics (NCBI), containing a large amount of data from microarrays, gene chips, and RNA-seq(Riksen \& Stienstra 2018). To obtain the genes associated with the formation as well as the development of atherosclerosis, the dataset consisted of human atherosclerotic plaques and normal arteries or early and advanced atherosclerotic plaques that were searched in the GEO database, and two datasets (GSE28829 and GSE43292) were obtained. Then, the invitro dataset contained ox-LDL treated smooth muscle cells (SMC) or macrophages that were screened out to identify foam cell-related DEGs of the two cell types. Furthermore, because of the important role of foam cells in the development of vulnerable plaques(Liu et al. 2017), the dataset composed of unstable vs. stable or ruptured vs. unruptured atherosclerotic plaques were screened to explore the expression of the foam cell-related genes in vulnerable plaques. The datasets of animal samples and the in-vivo human datasets of serum or plasma were excluded in this study.

The GSE28829(Döring et al. 2012) dataset contains 13 early carotid atherosclerotic plaque samples and 16 advanced atherosclerotic plaque samples. GSE43292(Ayari \& Bricca 2013) contains 32 normal carotid artery samples and 32 corresponding atherosclerotic plaque samples. GSE68021(Damián-Zamacona et al. 2016) contains the gene expression dataset of human vascular SMCs simulated with oxidized low-density lipoprotein (ox-LDL) for $0 \mathrm{~h}, 1 \mathrm{~h}, 5 \mathrm{~h}$, and $24 \mathrm{~h}$ (3 technical replicates of each group). GSE54666(Reschen et al. 2015) is a dataset of in vitro macrophage experiments including 6 samples of untreated macrophages and 6 samples of macrophage-derived FCs stimulated with ox-LDL for 48 h. GSE9874(Hägg et al. 2008) consists of the gene expression profiles of macrophages treated or untreated with ox-LDL from 15 healthy subjects and 15 atherosclerotic patients. GSE41571(Lee et al. 2013) contains data on 11 
127

128

129

130

131

132

133

134

135

136

137

138

139

140

141

142

143

144

145

146

147

macrophage-rich regions from 5 ruptured plaques and 6 stable plaques. GSE120521(Mahmoud et al. 2019) consists of an RNA-seq profile of 4 stable and 4 unstable plaque samples. The analysis strategy is shown in Figure 1.

\section{Identification of DEGs}

The downloaded gene expression profiles and their matched platform files were loaded into $\mathrm{R}$ (version 3.6.1) software and converted into gene symbol expression profiles. The LIMMA package was used to identify differentially expressed genes (DEGs) between the two groups(Ritchie et al. 2015). DEGs with adjusted $p$ values $<0.05$ (adjusted by the BenjaminiHochberg method) were considered significant. Co-DEGs were found in the overlap of different datasets as determined by an online web tool (http://jvenn.toulouse.inra.fr/app/example.html).

For the GSE68021 dataset, the DEGs of each time point (ox-LDL treated for $1 \mathrm{~h}, 5 \mathrm{~h}, 24 \mathrm{~h}$ ) compared with negative control were identified. Furthermore, the Short Time-series Expression Miner (STEM)(Ernst \& Bar-Joseph 2006) program was used to analyze the temporal expression profiles of these DEGs. The significant clusters which manifested upregulated or downregulated characteristics over time were selected for the next analysis.

\section{Functional enrichment analyses}

The purpose of gene function enrichment analysis is to determine the correlation between a group of genes and functional categories through a hypergeometric test. Gene Ontology (GO) is an international standardized gene functional classification system that covers three major 
148

149

150

151

152

categories: biological process (BP), cellular component (CC), and molecular function (MF). The Kyoto Encyclopedia of Genes and Genomes (KEGG) database is the major public pathwayrelated database for helping researchers understand the advanced functions of genes in biological systems. DAVID (https://david.ncifcrf.gov/home.jsp) is a very useful bioinformatics data resource for scientists to find meaningful biological information on genes or proteins(Huang da et al. 2009). Herein, GO-BP and KEGG pathway analyses were performed using the DAVID database. The functional category with a p-value $<0.05$ (Xia et al. 2019) was considered significant.

\section{Validation of the common FCs-related genes}

For the validation of the common FCs-related genes, the expressions of these genes were extracted out from the microarray dataset and analyzed by student's $t$-test. For the RNA-seq dataset, the normalized gene expression matrix of FPKM (fragments per kilobase of exon model per million reads mapped) values was downloaded and transformed into TPM (transcripts per kilobase million) values for comparison( $\mathrm{Li}$ et al. 2010). P value $<0.05$ was defined as statistical significance.

\section{Results}

\section{Identification of DEGs associated with atherosclerosis}

GSE28829 contained data on 13 early atherosclerotic plaques and 16 late atherosclerotic plaque samples. Through a LIMMA analysis, a total of 2,300 DEGs were identified in the advanced plaques, based on the gene expression in the early plaques (defined as atherosclerosis 
169

170

171

172

173

174

175

176

177

178

179

180

181

182

183

184

185

186

187

progression-related genes), including 1,150 upregulated genes and 1,150 downregulated genes

(Figure 2A). GSE43292 contained information on 32 nonatherosclerotic plaque samples

(control) and 32 atherosclerotic plaque samples, and 6,378 DEGs were obtained, including 3,066

up- and 3,312 downregulated DEGs in atherosclerosis, based on the gene expression in the

control group (Figure 2B). After comparing the DEGs in the two datasets, 801 co-

downregulated and 849 co-upregulated genes were identified (Figure 2C, D). These co-

regulated DEGs were defined as atherosclerosis-related genes.

\section{Identification of SMC-FCs-related genes and functional analysis}

GSE68021 includes the gene expression data of human vascular SMCs treated with ox-LDL

for $1 \mathrm{~h}, 5 \mathrm{~h}$, and $24 \mathrm{~h}$ in vitro to simulate the status of SMC-FCs in atheroma plaques. In this

study, a total of 3369, 4887, and 7170 DEGs were identified in 1 h, 5 h, and 24 h compared with

control (Figure 3A). Moreover, these DEGs obtained from different time points were

investigated their expressive trends by using STEM program. As shown in Figure 3B, 13 clusters

with statistical significance were identified. And Cluster 1 (581 genes), 9 (601 genes), 12 (392

genes), 11 (413 genes), 26 (253 genes), 23 (240 genes) exhibited downregulated features over

time, while Cluster 42 (630 genes), 48 (570 genes), 40 (493 genes), 29 (344 genes) exhibited

upregulated trends over time. The expression of these genes with downregulated and upregulated

trends were shown in Figure 3C. Then these genes were compared with AS-related genes, and a

total of 432 common genes were obtained and defined as SMC-FCs-related genes (Figure 3D).

To clarify the biological functions of these SMC-FCs-related genes, the GO-BP and KEGG 
190

191

192

193

pathway enrichment analyses were performed, and a total of 71 BP and 25 KEGG pathway functions were enriched. As shown in Figure 3E and supplementary Table S1, the enriched KEGG pathways were mainly involved in inflammation and contractive function pathways, such as Regulation of actin cytoskeleton (hsa04810), Vascular smooth muscle contraction (hsa04270), Chemokine signaling pathway (hsa04062), Leukocyte transendothelial migration (hsa04670). Similarly, the results of GO-BP terms suggested these genes were mainly involved in the contraction function, inflammation, cell cycle/apoptosis, and substance uptake and intracellular transport, such as actin filament organization (GO:0007015), smooth muscle contraction (GO:0006939), $\mathrm{T}$ cell receptor signaling pathway (GO:0050852), inflammatory response (GO:0006954), apoptotic process (GO:0006915), cell cycle (GO:0007049), early endosome to late endosome transport (GO:0045022), lysosome organization (GO:0007040) (Figure 3F and supplementary Table S1).

\section{Identification of DEGs associated with macrophage-derived foam cells (M-FCs) and their}

\section{functional enrichment}

GSE54666 was the gene expression profiling of macrophages stimulated with ox-LDL in vitro to simulate the status of M-FCs in atheroma plaques. A total of 484 differentially expressed genes were obtained, including 259 upregulated genes and 225 downregulated genes (Figure

4A). These DEGs and previously obtained AS-related genes were integrated, and the overlapping genes were defined as M-FC-related genes. As shown in Figure 4B, a total of 81 M-FC-related genes were identified. 
To explore the biological role of the $81 \mathrm{M}-\mathrm{FCs}-$ related genes, functional enrichment

212

213

214

215

216

217

218

219

220

221

222

223

224

225

226

227

228

229

230

231

analysis was performed again using the DAVID database. A total of 30 GO-BP and 1 KEGG

pathway were enriched. The GO-BP analysis mainly revealed lipid metabolism, foam cell

differentiation, and immune response, such as negative regulation of cholesterol storage

(GO:0010887), negative regulation of macrophage-derived foam cell differentiation

(GO:0010745), response to low-density lipoprotein particle (GO:0055098), and negative

regulation of interferon-gamma-mediated signaling pathway (GO:0060336). The enriched

KEGG pathway was the PPAR signaling pathway (hsa03320) (Figure 4C and Supplementary

Table S2).

\section{The common genes of SMC-FCs and M-FCs related DEGs}

To identify common molecular mechanisms in the SMC-FCs and M-FCs, the common genes

were screened out. As shown in Figure 5A, 15 common genes were identified: CTSD, HHEX,

TNFRSF21, GLRX, EDEM2, CTSC, LAT2, SPOCD1, ABCA1, LST1, CD74, PLAUR, BRI3,

$E M B$, and $R N F 13$. Then, the expression trends of these 15 genes in AS tissue, SMC-FCs, and M-

FCs were compared to that of the respective control. As shown in Figure 5 B-C, all of these 15

genes were upregulated in the atherosclerotic group compared to the non-atherosclerotic group or

in advanced plaques compared with early plaques. However, only four genes ( $G L R X, A B C A 1$,

$H H E X$, and RNF13) exhibited upregulated trends in ox-LDL treated SMCs (Figure 5D), while

nine genes (SPOCD1, PLAUR, CTSD, RNF13, ABCA1, BRI3, GLRX, TNFRSF21, and EDEM2)

were increased in ox-LDL treated macrophages (Figure 5E). Then, the co-upregulated and co- 
232 downregulated genes were screened. The Venn diagrams showed the 3 (GLRX, RNF13, and $233 A B C A 1)$ co-upregulated genes; and no co-downregulated genes were found (Figure 5F, G).

Validation of the common FCs-related genes and their expression in vulnerable plaques

The expression of the 3 co-upregulated FC genes was then validated using another dataset.

GSE9874 contains the gene expression profile of peripheral blood monocyte-derived macrophages treated or untreated with ox-LDL. As shown in Figure 6A, all of the three genes were increased in the ox-LDL treated group (Foam cells group).

Previous studies have suggested that the formation and retention of the FCs could exacerbate atherosclerosis and fuel the development of the vulnerable plaques(Bäck \& Hansson 2015; Liu et al. 2017). Therefore, the expressions of the three FCs-related genes were investigated in the vulnerable plaques. As shown in Figure 6B, only RNF13 was statistically significantly upregulated in unstable plaques, while $A B C A 1$ and GLRX showed no statistical significance, though the means of $A B C A 1$ were larger in unstable plaques than stable plaques. In ruptured plaques, GLRX and $R N F 13$ were significantly upregulated compared with stable plaques, while $A B C A 1$ showed a slight increase with no statistical significance (Figure 6C).

\section{Discussion}

Previous studies have suggested the crucial role of foam cells (FCs) in the formation and development of atherosclerosis, and the molecular mechanisms of FCs have been the focus of 
253

254

256

257

258

259

260

261

262

263

264

265

266

267

268

269

270

271

272

273

throughput and bioinformatic technologies have been widely used for exploring the molecular

mechanisms and predicting biomarkers for the diagnosis, treatment, and prognosis of the diseases(Dona et al. 2016). Through the gene expression microarray technology, Döring Y et al. revealed the upregulation of the plasmacytoid dendritic cell markers (such as CD123, CD83, E22, $C D 317, C D 32$, and $B D C A 2$ ) in advanced versus early lesions(Döring et al. 2012). Ayari $\mathrm{H}$ et al. identified two genes (CDI63 and $\mathrm{HO}-1)$, which were significantly increased in atheroma plaques compared with intact arterial tissue by using GeneChip arrays(Ayari \& Bricca 2013).

Lee $\mathrm{K}$ et al. identified $914 \mathrm{DEGs}$ between stable and ruptured plaques by using microarray technology, and highlighted the involvement of FABP4 and leptin in the progression of atherosclerosis and plaque rupture(Lee et al. 2013). Mahmoud AD et al. revealed the DEGs between stable and unstable plaques, which were mainly linked with the plaque instability, including inflammation, matrix remodeling, and calcification(Mahmoud et al. 2019). Although

these studies revealed some molecular mechanisms of the atherosclerosis development in various aspects, they could not reveal the cell-specific mechanisms, such as foam cells. Therefore, some studies tried to investigate the molecular changes of specific cells by in-vitro experiments, such as macrophages and SMCs under the stimulation of ox-LDL(Damián-Zamacona et al. 2016; Hägg et al. 2008; Reschen et al. 2015). However, ox-LDL treatment alone might be much too oversimplified because of the complicated environment of cells in organisms. Therefore, in this study, we tried to combine the in-vitro and in-vivo experiments to relieve these influences.

In the present study, two datasets of gene expression in atherosclerosis tissues (GSE28829 and GSE43292) were analyzed, and 1650 co-regulated DEGs were identified. After screening 
274

275

276

277

278

279

280

281

282

283

284

285

286

287

288

289

290

291

292

293

294

these 1650 co-DEGs with DEGs of SMC-FCs (GSE68021) and M-FCs (GSE54666), a total of

432 SMC-FCs-related genes and 81 M-FCs-related genes were identified. To identify the

common therapeutic targets of FCs, the common molecular mechanism between FCs derived

from SMCs and macrophages was explored. Three co-upregulated genes were identified: GLRX,

$R N F 13$, and $A B C A 1$. Furthermore, the expressions of these 3 genes in vulnerable atherosclerosis

plaques were explored, and all of them were increasingly expressed in unstable or ruptured

plaques compared to their expression levels in stable plaques, although some of the increased

levels were not statistically significant.

Based on murine models, it was long presumed that all FCs in human atherosclerosis were

derived from macrophages. However, subsequent studies demonstrated that resident cell types,

especially SMCs, maybe the prominent originators of FCs(Poznyak et al. 2020; Wang et al.

2019b). However, macrophages are still the second most prolific sources of FCs and play crucial

roles in the pathogenesis of atherosclerosis, in both the early and advanced stages(Owsiany et al.

2019). Therefore, macrophages remain the most representative model of FCs and will continue

to be studied.

The process of lipid metabolism in macrophages is divided into three main stages: lipid

uptake, esterification, and efflux(Maguire et al. 2019). The lipid metabolism of macrophages in

atheroma plaques gradually accelerates to be imbalanced, with excessive intracellular lipid

deposition and leading to the formation of FCs(Chistiakov et al. 2017; Maguire et al. 2019). In

the present study, a total of $81 \mathrm{M}-\mathrm{FC}$-related genes were identified, and their functions were

found to be mainly enriched in lipid metabolism and immune responses.

PeerJ reviewing PDF | (2020:06:50248:2:0:NEW 14 Oct 2020) 

transporters that depend on multiple key transcription factors, such as PPAR and

LXLR(Chistiakov et al. 2016; Chistiakov et al. 2017). These transporters mainly include ATP-

binding cassette transporter 1 (ABCA1), ATP-binding cassette subfamily member G1 (ABCG1), and SR-B1, which play crucial roles in preventing the excessive intracellular accumulation of cholesterol and the formation of FCs(DiMarco \& Fernandez 2015). Similar to that of cholesterol internalization, a confusing relationship characterizes these efflux transporters during the progression of atherosclerosis. For example, a knockout of ABCA1 aggravated the formation of

FCs but did not promote atherosclerosis plaque development(Zhao et al. 2011). Moreover,

studies of ABCG1 and SR-B1 have also revealed controversial results, as they have a promoting role in early atherogenesis but a protecting role in advanced atherosclerosis(Meurs et al. 2012;

Van Eck et al. 2004; Zhang et al. 2003).

SMCs also play crucial roles in all stages of atherosclerosis, and they are considered the leading sources of FCs(Poznyak et al. 2020; Wang et al. 2019b). During the process of atherosclerosis, activated macrophages may secrete many inflammatory factors or cytokines that can promote the phenotypic transformation of SMCs from the resting systolic phenotype to the activated synthetic or "macrophage-like" phenotype. These phenotypic changes in SMCs are generally associated with the downregulated expression of contractile proteins and increased proliferation(Zhang et al. 2012). In the present study, a total of 432 SMC-FC-related genes were identified, and they were mainly involved in contraction function, inflammation, cell 
316

atherogenesis. Moreover, compared with M-FCs, the expression of the cholesterol efflux transporter ABCA1 in SMC-FCs is much lower. Furthermore, the ABCA1 level in SMCs in advanced atherosclerosis was decreased compared to early atherosclerosis; however, no changes in macrophages were observed(Allahverdian et al. 2014). These results provide a plausible explanation for the high percentage of FCs derived from SMCs(Allahverdian et al. 2014). RNF13 is an E3 ubiquitin ligase that is widely expressed in various animals and tissues. Previous studies have determined the roles of RNF13 in myogenesis, neuronal development, and tumorigenesis(Zhang et al. 2009; Zhang et al. 2010), in which it is mainly involved in cell proliferation(Jin et al. 2011). But few studies investigated the role of RNF13 in atherosclerosis or FCs. GLRX is a member of the glutaredoxin family, which highly contributes to the antioxidant defense system. Overexpression of GLRX could attenuate H2O2-induced apoptosis of endothelial cells(Li et al. 2017). However, GLRX inhibits endothelial cell angiogenic properties(Matsui et al. 2017). Though increasing evidence has suggested the important roles of oxidative stress in atherosclerosis(Sharif et al. 2020; Zhang et al. 2020), few studies have revealed the role of GLRX in atherosclerosis and the formation of FCs. Therefore, the roles of RNF13 and GLRX in the process of atherosclersis and FCs remain unknown, which needs further exploration.

The present study has several limitations. Firstly, the sample size of some datasets used in this study is small, which might result in some bias. For example, the GSE120521 contained only 4 stable and 4 unstable samples. Secondly, there may be some confounding factors, such as types and morphology of atherosclerotic plaques, age, gender, and drug treatment, as well as clinical 
337

complications, which might affect the interpretation of the results, and could not be identified in

this study. Thirdly, interexperiment variability, such as different detection platforms and different resources of samples from different laboratories, may result in somewhat differences in gene expressions. Moreover, different cell types, like SMCs and macrophages, might show different changes of gene expression in ox-LDL treatment or pro-atheromatous environments in tissues. Therefore, only three co-up regulated genes were identified in this study between the two cell types of foam cells.

\section{Conclusion}

In the present study, 432 SMC-FC-related genes and $81 \mathrm{M}-F C$-related genes were identified, and they were found to be mainly involved in lipid metabolism, inflammation, cell cycle/apoptosis. Furthermore, 3 co-regulated genes associated with FCs were identified: GLRX, $R N F 13$, and $A B C A 1$. Among these genes, the roles of $A B C A 1$ in the atherosclerotic process have been widely described, but many contradictory results have been presented, suggesting that the roles of $A B C A 1$ in atherosclerosis need to be further explored. Moreover, the roles of $R N F 13$ and GLRX in atherogenesis remain unknown, and more experiments in the future are needed to confirm their roles. These three genes have a common expression tendency in both SMCs and macrophages, which may have implications for the development of possible targeted therapeutic drugs in the future.

\section{Acknowledgements}


None.

\section{Reference}

Allahverdian S, Chehroudi AC, McManus BM, Abraham T, and Francis GA. 2014. Contribution of intimal smooth muscle cells to cholesterol accumulation and macrophage-like cells in human atherosclerosis. Circulation 129:1551-1559. 10.1161/circulationaha.113.005015

Ayari H, and Bricca G. 2013. Identification of two genes potentially associated in iron-heme homeostasis in human carotid plaque using microarray analysis. J Biosci 38:311-315. 10.1007/s12038-013-9310-2

Bäck M, and Hansson GK. 2015. Anti-inflammatory therapies for atherosclerosis. Nat Rev Cardiol 12:199-211. 10.1038/nrcardio.2015.5

Chistiakov DA, Bobryshev YV, and Orekhov AN. 2016. Macrophage-mediated cholesterol handling in atherosclerosis. J Cell Mol Med 20:17-28. 10.1111/jcmm.12689

Chistiakov DA, Melnichenko AA, Myasoedova VA, Grechko AV, and Orekhov AN. 2017. Mechanisms of foam cell formation in atherosclerosis. $J$ Mol Med (Berl) 95:1153-1165. 10.1007/s00109-017-1575-8

Damián-Zamacona S, Toledo-Ibelles P, Ibarra-Abundis MZ, Uribe-Figueroa L, Hernández-Lemus E, Macedo-Alcibia KP, Delgado-Coello B, Mas-Oliva J, and Reyes-Grajeda JP. 2016. Early Transcriptomic Response to LDL and oxLDL in Human Vascular Smooth Muscle Cells. PLoS One 11:e0163924. 10.1371/journal.pone.0163924

DiMarco DM, and Fernandez ML. 2015. The Regulation of Reverse Cholesterol Transport and Cellular Cholesterol Homeostasis by MicroRNAs. Biology (Basel) 4:494-511. 10.3390/biology4030494

Dona AC, Coffey S, and Figtree G. 2016. Translational and emerging clinical applications of metabolomics in cardiovascular disease diagnosis and treatment. Eur $J$ Prev Cardiol 23:1578-1589. $10.1177 / 2047487316645469$

Döring Y, Manthey HD, Drechsler M, Lievens D, Megens RT, Soehnlein O, Busch M, Manca M, Koenen RR, Pelisek J, Daemen MJ, Lutgens E, Zenke M, Binder CJ, Weber C, and Zernecke A. 2012. Auto-antigenic proteinDNA complexes stimulate plasmacytoid dendritic cells to promote atherosclerosis. Circulation 125:16731683. 10.1161/circulationaha.111.046755

Ernst J, and Bar-Joseph Z. 2006. STEM: a tool for the analysis of short time series gene expression data. $B M C$ Bioinformatics 7:191. 10.1186/1471-2105-7-191

Gisterå A, and Hansson GK. 2017. The immunology of atherosclerosis. Nat Rev Nephrol 13:368-380. 10.1038/nrneph.2017.51

Hägg DA, Jernås M, Wiklund O, Thelle DS, Fagerberg B, Eriksson P, Hamsten A, Olsson B, Carlsson B, Carlsson LM, and Svensson PA. 2008. Expression profiling of macrophages from subjects with atherosclerosis to identify novel susceptibility genes. Int J Mol Med 21:697-704.

Huang da W, Sherman BT, and Lempicki RA. 2009. Systematic and integrative analysis of large gene lists using DAVID bioinformatics resources. Nat Protoc 4:44-57. 10.1038/nprot.2008.211

Huang HM, Jiang X, Hao ML, Shan MJ, Qiu Y, Hu GF, Wang Q, Yu ZQ, Meng LB, and Zou YY. 2019. Identification of biomarkers in macrophages of atherosclerosis by microarray analysis. Lipids Health Dis 18:107. 


\subsection{6/s12944-019-1056-X}

Jin X, Cheng H, Chen J, and Zhu D. 2011. RNF13: an emerging RING finger ubiquitin ligase important in cell proliferation. Febs j 278:78-84. 10.1111/j.1742-4658.2010.07925.x

Kanter JE, Kramer F, Barnhart S, Duggan JM, Shimizu-Albergine M, Kothari V, Chait A, Bouman SD, Hamerman JA, Hansen BF, Olsen GS, and Bornfeldt KE. 2018. A Novel Strategy to Prevent Advanced Atherosclerosis and Lower Blood Glucose in a Mouse Model of Metabolic Syndrome. Diabetes 67:946-959. 10.2337/db170744

Katakami N. 2018. Mechanism of Development of Atherosclerosis and Cardiovascular Disease in Diabetes Mellitus. $J$ Atheroscler Thromb 25:27-39. 10.5551/jat.RV17014

Lee K, Santibanez-Koref M, Polvikoski T, Birchall D, Mendelow AD, and Keavney B. 2013. Increased expression of fatty acid binding protein 4 and leptin in resident macrophages characterises atherosclerotic plaque rupture. Atherosclerosis 226:74-81. 10.1016/j.atherosclerosis.2012.09.037

Li B, Ruotti V, Stewart RM, Thomson JA, and Dewey CN. 2010. RNA-Seq gene expression estimation with read mapping uncertainty. Bioinformatics 26:493-500. 10.1093/bioinformatics/btp692

Li Y, Ren M, Wang X, Cui X, Zhao H, Zhao C, Zhou J, Guo Y, Hu Y, Yan C, Berk B, and Wang J. 2017. Glutaredoxin 1 mediates the protective effect of steady laminar flow on endothelial cells against oxidative stress-induced apoptosis via inhibiting Bim. Sci Rep 7:15539. 10.1038/s41598-017-15672-3

Liu Y, Huan W, Wu J, Zou S, and Qu L. 2020. IGFBP6 Is Downregulated in Unstable Carotid Atherosclerotic Plaques According to an Integrated Bioinformatics Analysis and Experimental Verification. J Atheroscler Thromb. $10.5551 /$ jat. 52993

Liu Z, Zhu H, Dai X, Wang C, Ding Y, Song P, and Zou MH. 2017. Macrophage Liver Kinase B1 Inhibits Foam Cell Formation and Atherosclerosis. Circ Res 121:1047-1057. 10.1161/circresaha.117.311546

Maguire EM, Pearce SWA, and Xiao Q. 2019. Foam cell formation: A new target for fighting atherosclerosis and cardiovascular disease. Vascul Pharmacol 112:54-71. 10.1016/j.vph.2018.08.002

Mahmoud AD, Ballantyne MD, Miscianinov V, Pinel K, Hung J, Scanlon JP, Iyinikkel J, Kaczynski J, Tavares AS, Bradshaw AC, Mills NL, Newby DE, Caporali A, Gould GW, George SJ, Ulitsky I, Sluimer JC, Rodor J, and Baker AH. 2019. The Human-Specific and Smooth Muscle Cell-Enriched LncRNA SMILR Promotes Proliferation by Regulating Mitotic CENPF mRNA and Drives Cell-Cycle Progression Which Can Be Targeted to Limit Vascular Remodeling. Circ Res 125:535-551. 10.1161/circresaha.119.314876

Matsui R, Watanabe Y, and Murdoch CE. 2017. Redox regulation of ischemic limb neovascularization - What we have learned from animal studies. Redox Biol 12:1011-1019. 10.1016/j.redox.2017.04.040

Meurs I, Lammers B, Zhao Y, Out R, Hildebrand RB, Hoekstra M, Van Berkel TJ, and Van Eck M. 2012. The effect of ABCG1 deficiency on atherosclerotic lesion development in LDL receptor knockout mice depends on the stage of atherogenesis. Atherosclerosis 221:41-47. 10.1016/j.atherosclerosis.2011.11.024

Olivares AL, González Ballester MA, and Noailly J. 2016. Virtual exploration of early stage atherosclerosis. Bioinformatics 32:3798-3806. 10.1093/bioinformatics/btw551

Owsiany KM, Alencar GF, and Owens GK. 2019. Revealing the Origins of Foam Cells in Atherosclerotic Lesions. Arterioscler Thromb Vasc Biol 39:836-838. 10.1161/atvbaha.119.312557

Poznyak AV, Wu WK, Melnichenko AA, Wetzker R, Sukhorukov V, Markin AM, Khotina VA, and Orekhov AN. 2020. Signaling Pathways and Key Genes Involved in Regulation of foam Cell Formation in Atherosclerosis. Cells 9. 10.3390/cells9030584 
437

438

439

440

441

442

443

444

445

446

447

448

449

450

451

452

453

454

455

456

457

458

459

460

461

462

463

464

465

466

467

468

469

470

471

472

473

474

475

476

477

Reschen ME, Gaulton KJ, Lin D, Soilleux EJ, Morris AJ, Smyth SS, and O'Callaghan CA. 2015. Lipid-induced epigenomic changes in human macrophages identify a coronary artery disease-associated variant that regulates PPAP2B Expression through Altered C/EBP-beta binding. PLoS Genet 11:e1005061. 10.1371/journal.pgen.1005061

Riksen NP, and Stienstra R. 2018. Metabolism of innate immune cells: impact on atherosclerosis. Curr Opin Lipidol 29:359-367. 10.1097/mol.0000000000000539

Ritchie ME, Phipson B, Wu D, Hu Y, Law CW, Shi W, and Smyth GK. 2015. limma powers differential expression analyses for RNA-sequencing and microarray studies. Nucleic Acids Res 43:e47. 10.1093/nar/gkv007

Sharif H, Akash MSH, Rehman K, Irshad K, and Imran I. 2020. Pathophysiology of atherosclerosis: Association of risk factors and treatment strategies using plant-based bioactive compounds. J Food Biochem:e13449. $10.1111 /$ jbc. 13449

Summerhill VI, Grechko AV, Yet SF, Sobenin IA, and Orekhov AN. 2019. The Atherogenic Role of Circulating Modified Lipids in Atherosclerosis. Int J Mol Sci 20. 10.3390/ijms20143561

Tan X, Zhang X, Pan L, Tian X, and Dong P. 2017. Identification of Key Pathways and Genes in Advanced Coronary Atherosclerosis Using Bioinformatics Analysis. Biomed Res Int 2017:4323496. 10.1155/2017/4323496

Van Eck M, Bos IS, Hildebrand RB, Van Rij BT, and Van Berkel TJ. 2004. Dual role for scavenger receptor class B, type I on bone marrow-derived cells in atherosclerotic lesion development. Am J Pathol 165:785-794. 10.1016/s0002-9440(10)63341-x

Vernon ST, Hansen T, Kott KA, Yang JY, O'Sullivan JF, and Figtree GA. 2019. Utilizing state-of-the-art "omics" technology and bioinformatics to identify new biological mechanisms and biomarkers for coronary artery disease. Microcirculation 26:e12488. 10.1111/micc.12488

Wang CH, Shi HH, Chen LH, Li XL, Cao GL, and Hu XF. 2019a. Identification of Key IncRNAs Associated With Atherosclerosis Progression Based on Public Datasets. Front Genet 10:123. 10.3389/fgene.2019.00123

Wang Y, Dubland JA, Allahverdian S, Asonye E, Sahin B, Jaw JE, Sin DD, Seidman MA, Leeper NJ, and Francis GA. 2019b. Smooth Muscle Cells Contribute the Majority of Foam Cells in ApoE (Apolipoprotein E)Deficient Mouse Atherosclerosis. Arterioscler Thromb Vasc Biol 39:876-887. 10.1161/atvbaha.119.312434

Weber C, and Noels H. 2011. Atherosclerosis: current pathogenesis and therapeutic options. Nat Med 17:1410-1422. $10.1038 / \mathrm{nm} .2538$

Xia WX, Yu Q, Li GH, Liu YW, Xiao FH, Yang LQ, Rahman ZU, Wang HT, and Kong QP. 2019. Identification of four hub genes associated with adrenocortical carcinoma progression by WGCNA. PeerJ 7:e6555. 10.7717/peerj.6555

Zhang H, Bai Z, Zhu L, Liang Y, Fan X, Li J, Wen H, Shi T, Zhao Q, and Wang Z. 2020. Hydrogen sulfide donors: Therapeutic potential in anti-atherosclerosis. Eur J Med Chem 205:112665. 10.1016/j.ejmech.2020.112665

Zhang Q, Meng Y, Zhang L, Chen J, and Zhu D. 2009. RNF13: a novel RING-type ubiquitin ligase over-expressed in pancreatic cancer. Cell Res 19:348-357. 10.1038/cr.2008.285

Zhang Q, Wang K, Zhang Y, Meng J, Yu F, Chen Y, and Zhu D. 2010. The myostatin-induced E3 ubiquitin ligase RNF13 negatively regulates the proliferation of chicken myoblasts. Febs $j$ 277:466-476. 10.1111/j.17424658.2009.07498.x

Zhang W, Yancey PG, Su YR, Babaev VR, Zhang Y, Fazio S, and Linton MF. 2003. Inactivation of macrophage scavenger receptor class B type I promotes atherosclerotic lesion development in apolipoprotein E-deficient mice. Circulation 108:2258-2263. 10.1161/01.Cir.0000093189.97429.9d

PeerJ reviewing PDF | (2020:06:50248:2:0:NEW 14 Oct 2020) 
Zhang Z, Wang M, Fan XH, Chen JH, Guan YY, and Tang YB. 2012. Upregulation of TRPM7 channels by angiotensin II triggers phenotypic switching of vascular smooth muscle cells of ascending aorta. Circ Res 111:1137-1146. 10.1161/circresaha.112.273755

Zhao Y, Pennings M, Vrins CL, Calpe-Berdiel L, Hoekstra M, Kruijt JK, Ottenhoff R, Hildebrand RB, van der Sluis R, Jessup W, Le Goff W, Chapman MJ, Huby T, Groen AK, Van Berkel TJ, and Van Eck M. 2011. Hypocholesterolemia, foam cell accumulation, but no atherosclerosis in mice lacking ABC-transporter A1 and scavenger receptor BI. Atherosclerosis 218:314-322. 10.1016/j.atherosclerosis.2011.07.096

Zhou J, Zhang C, Wu X, Xie Q, Li L, Chen Y, Yan H, Ren P, and Huang X. 2019. Identification of genes and pathways related to atherosclerosis comorbidity and depressive behavior via RNA-seq and bioinformation analysis in ApoE(-/-) mice. Ann Transl Med 7:733. 10.21037/atm.2019.11.118 


\section{Figure 1}

Flow chart of the analysis.

DEGs: differentially expressed genes; NC: negative control; hVSMCs: human vascular smooth muscle cells; ox-LDL: oxidized low-density lipoprotein; SMC-FCs: smooth muscle cell-derived foam cells; M-FCs: macrophage-derived foam cells; PPI: protein-protein interaction; GO: Gene Ontology; BP: biological process; and KEGG: Kyoto Encyclopedia of Genes and Genomes.

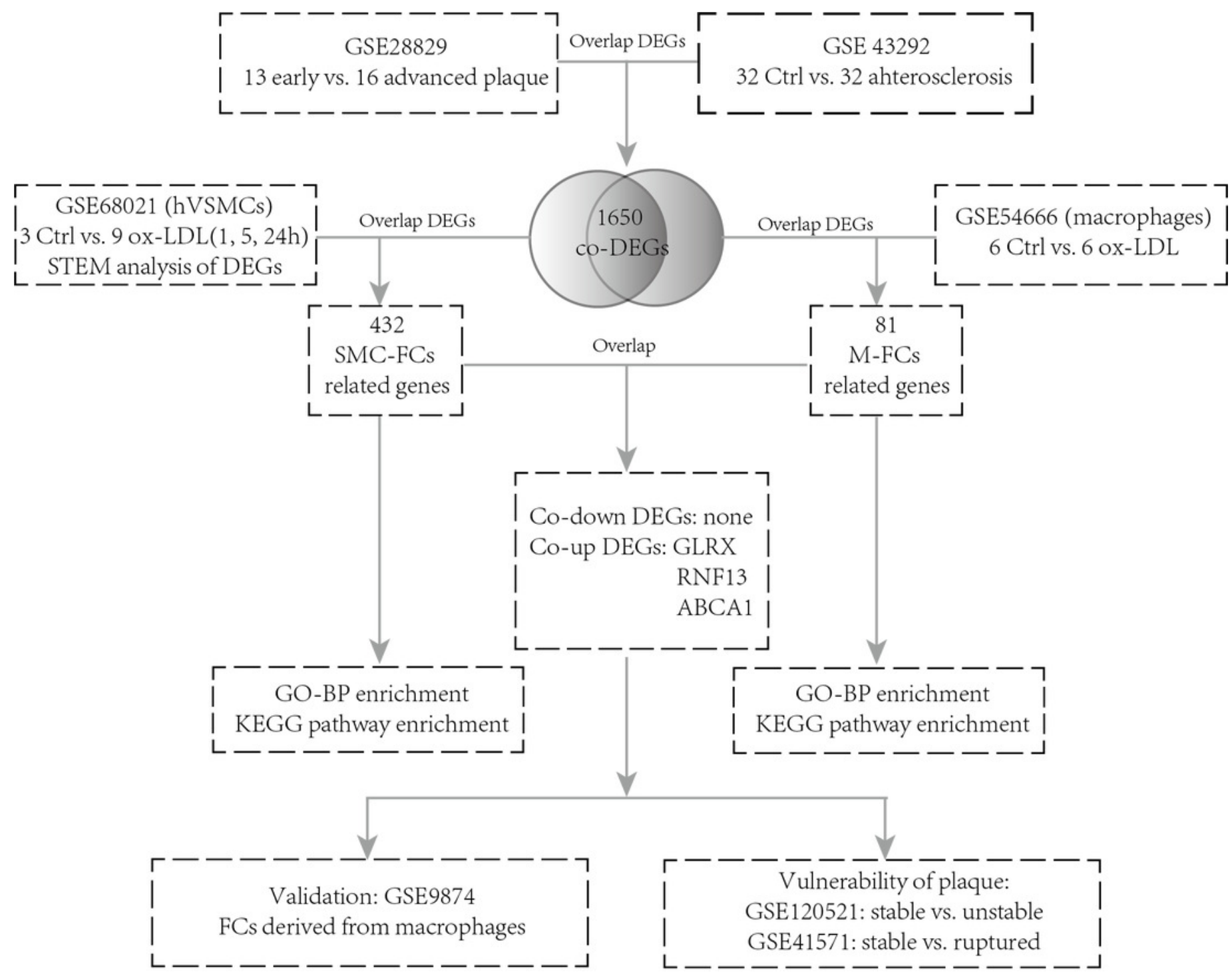




\section{Figure 2}

Identification of DEGs associated with atherosclerosis.

Hierarchical cluster heatmap of DEGs in advanced atherosclerosis compared to those in early atherosclerosis samples in the GSE28829 dataset (A) and those in atherosclerosis samples compared to those in non-atherosclerosis samples in the GSE43292 dataset (B). Venn diagrams showing the co-upregulated DEGs (C) and co-downregulated DEGs (D) in the GSE28829 and GSE43292 datasets, which were defined as atherosclerosis-related genes.

A

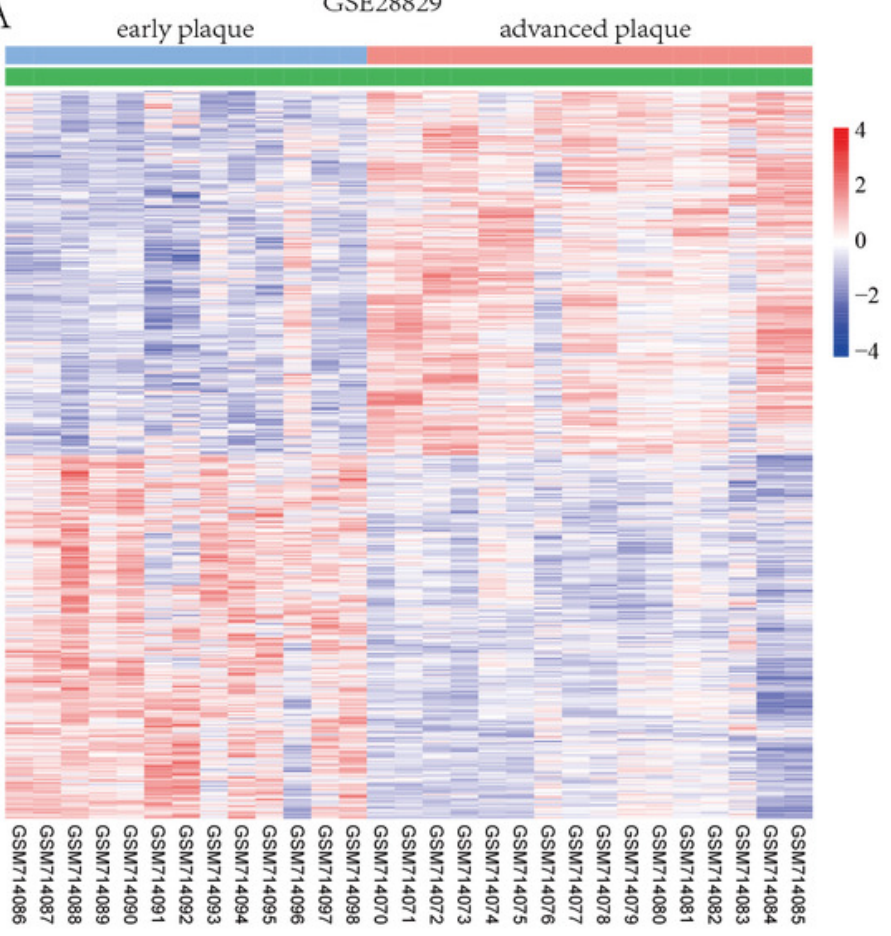

C

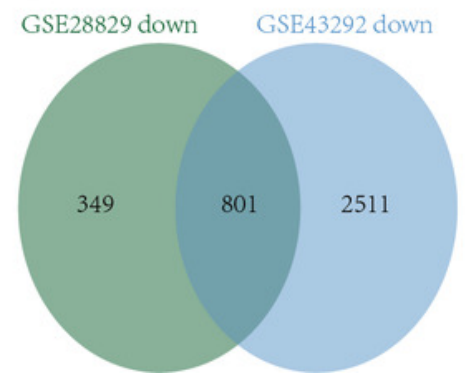

$\mathrm{B}$

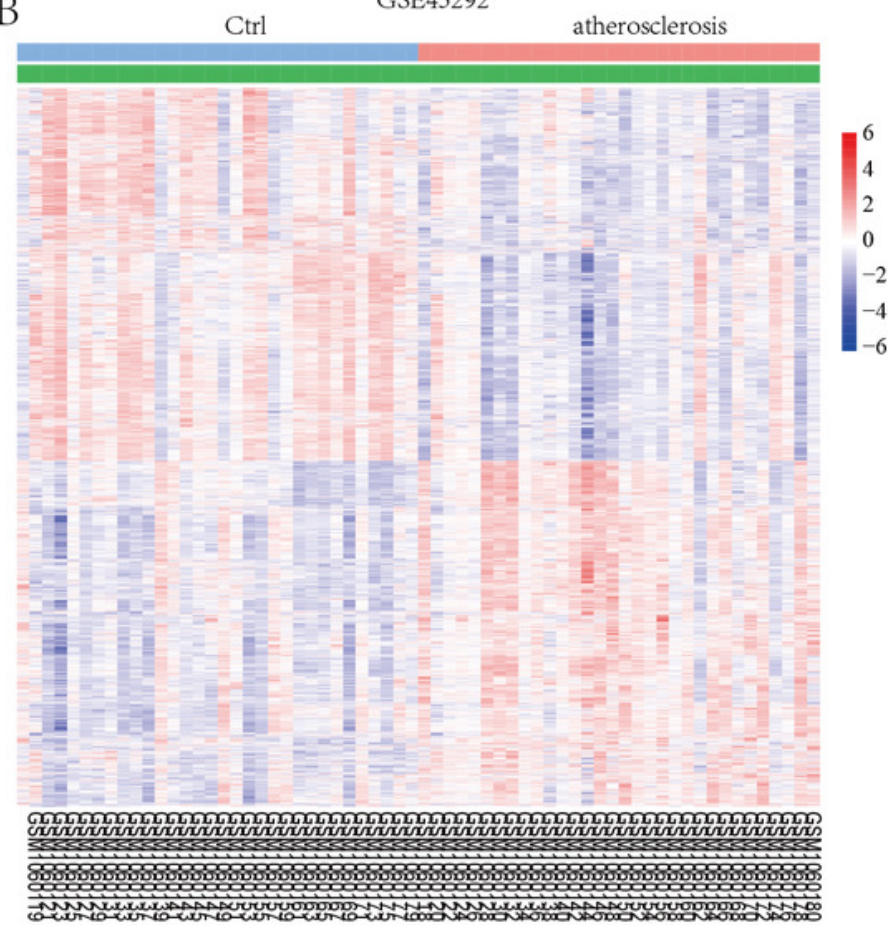

D

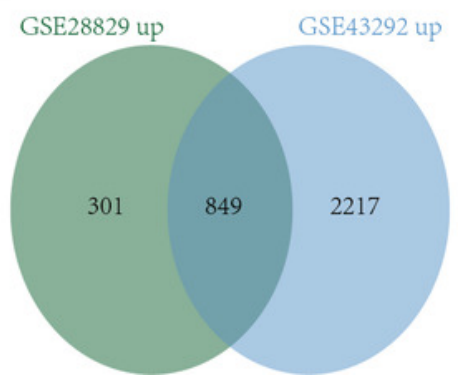




\section{Figure 3}

Identification of SMC-FCs-related genes and functional analysis.

(A) Venn diagram showing the DEGs of each time point (ox-LDL treated for $1 \mathrm{~h}, 5 \mathrm{~h}$, and 24

h) compared with control. (B) The expression trend clusters of all DEGs obtained above by

STEM analysis. (C) Hierarchical cluster heatmap of DEGs with downregulated and

upregulated trends overtime obtained by STEM analysis. (D) Venn diagram showing the overlap of DEGs in the ox-LDL-treated SMCs (with downregulated and upregulated trends overtime obtained by STEM analysis) and atherosclerosis-related genes, which were considered SMC-FCs-related DEGs. (E) and (F) The bubble diagram of the KEGG pathway and GO-BP enrichment analyses of SMC-FCs-related DEGs. In the bubble diagram, dot sizes represent counts of enriched DEGs, and dot colors represent negative $\log _{10}$ values ( $p$ values). SMC-FCs: smooth muscle cell derived foam cells; GO: Gene Ontology; BP: biological process; and KEGG: Kyoto Encyclopedia of Genes and Genomes; ox-LDL: oxidized low-density lipoprotein. 
A

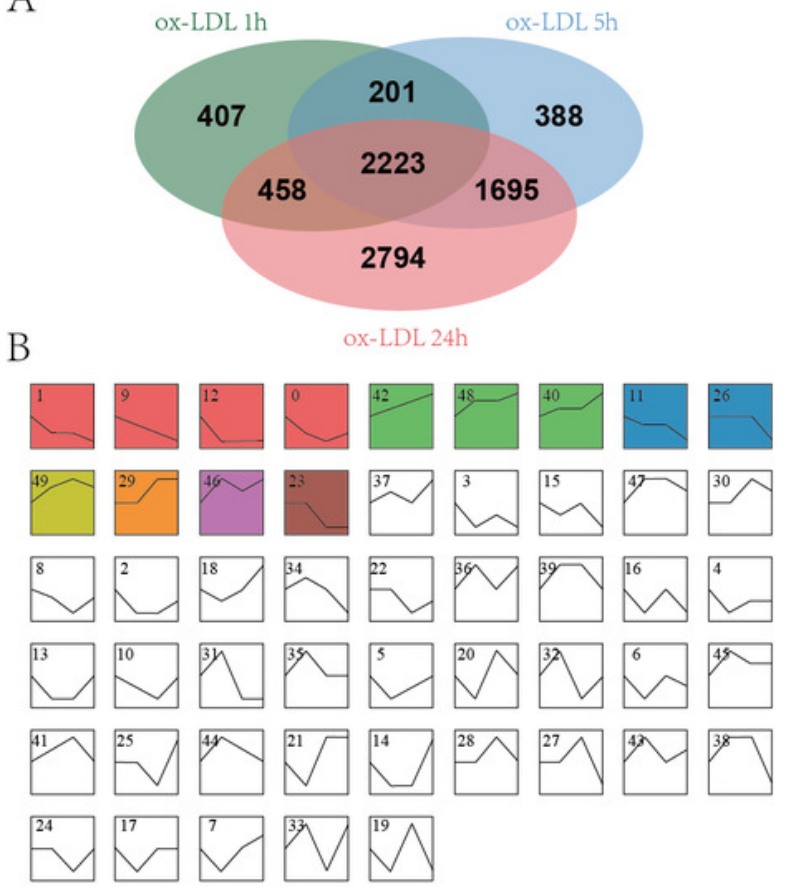

C

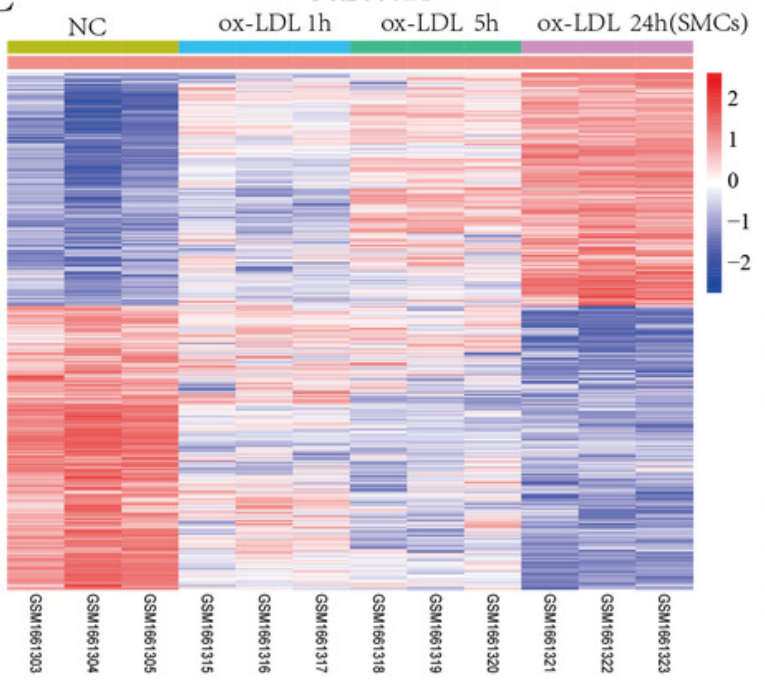

D atherosclerosis related genes

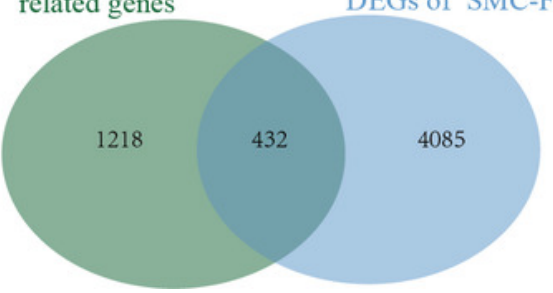

E

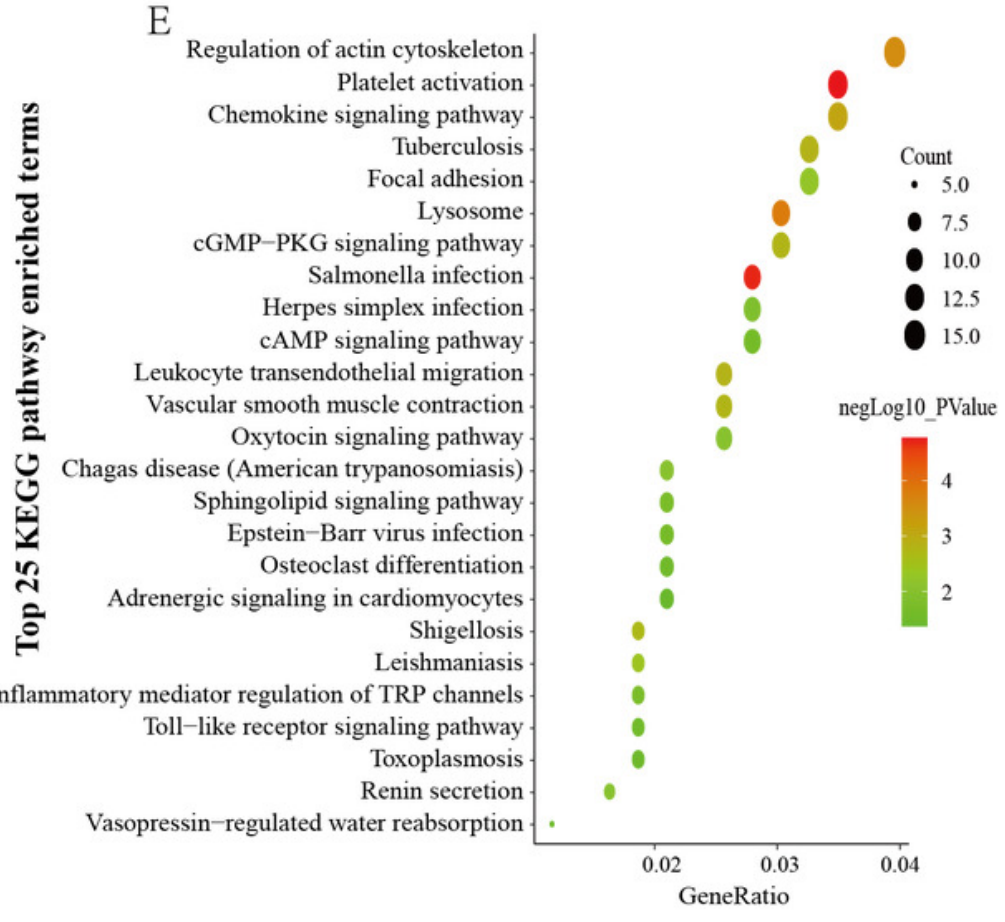

F

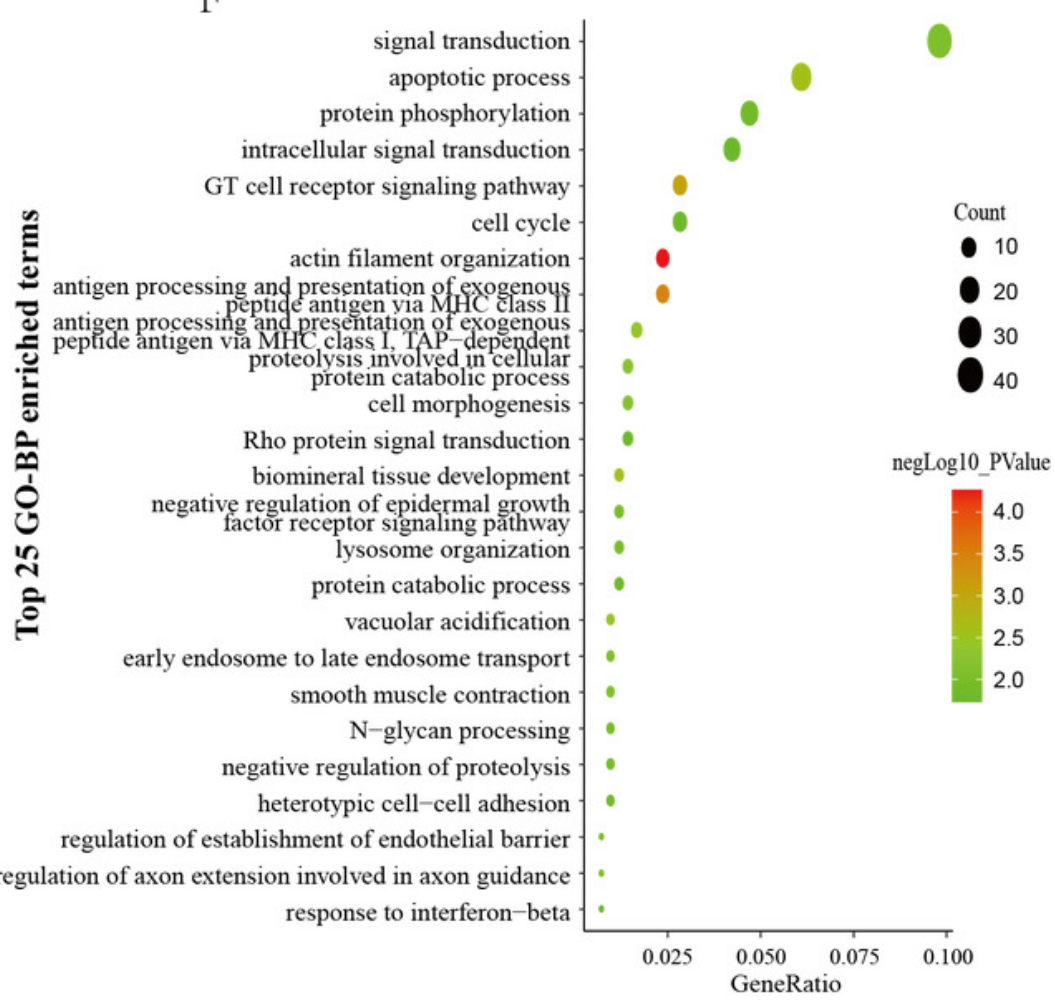




\section{Figure 4}

Identification of DEGs associated with M-FCs.

(A) Hierarchical cluster heatmap of the DEGs in the ox-LDL-stimulated macrophages compared to gene expression levels in the negative control from the GSE54666 dataset. Red represents upregulated genes, and green represents downregulated genes. (B) Venn diagram of the DEGs in the GSE54666 dataset compared to the AS-related genes and considered M-FC-related DEGs. (C) GO-BP and KEGG pathway enrichment analyses of the MFC-related DEGs. In the bubble diagram, dot sizes represent counts of enriched DEGs, and dot colors represent negative $\log _{10}$ values ( $p$ values). DEGs: differentially expressed genes; M-FCs: macrophage-derived foam cells; ox-LDL: oxidized low-density lipoprotein; AS: atherosclerosis; GO: Gene Ontology; BP: biological process; and KEGG: Kyoto Encyclopedia of Genes and Genomes. 
A

Ctrl

x-LDL macrophage

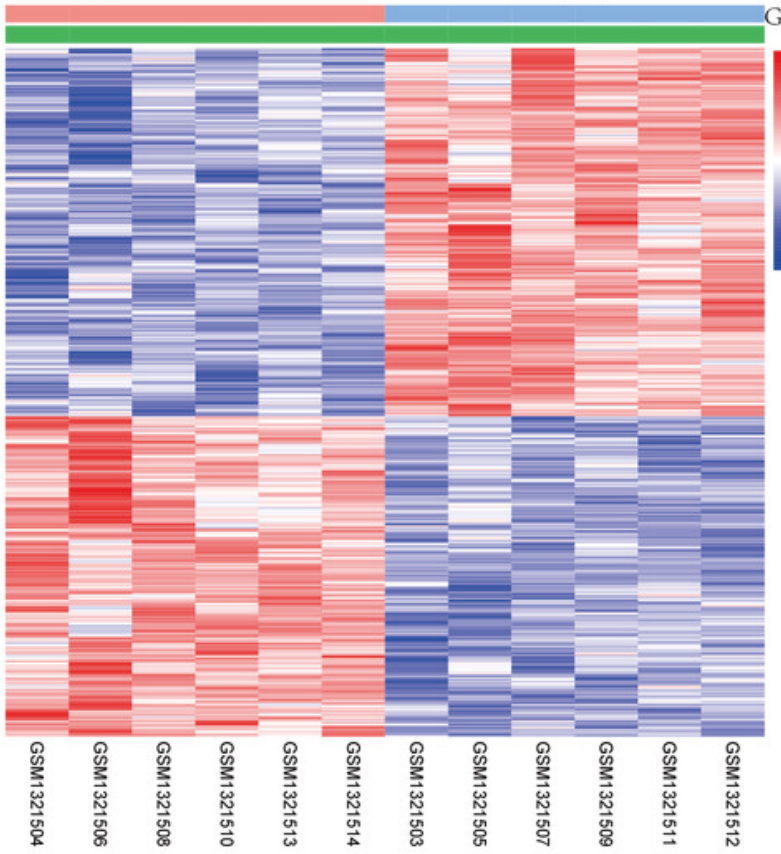

B

atherosclerosis related genes

EGs of M-FCs
C

2
1
0
-1
-2

है.

PPAR signaling pathwar

negative regulation of cholesterol storag.

response to low-density lipoprotein-

negative regulation of interferon-gamma negative regulation of interferon-gammated signaling pathway
mediate

है

tye regulation of macrophage

rentiation

(a)

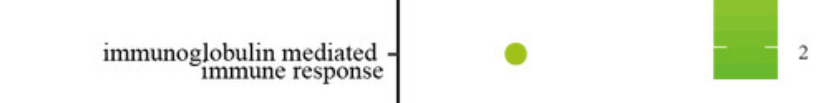

positive regulation of cholesterol efflut

cellular response to lipopolysaccharid

immune response

Count

- 4

8

.
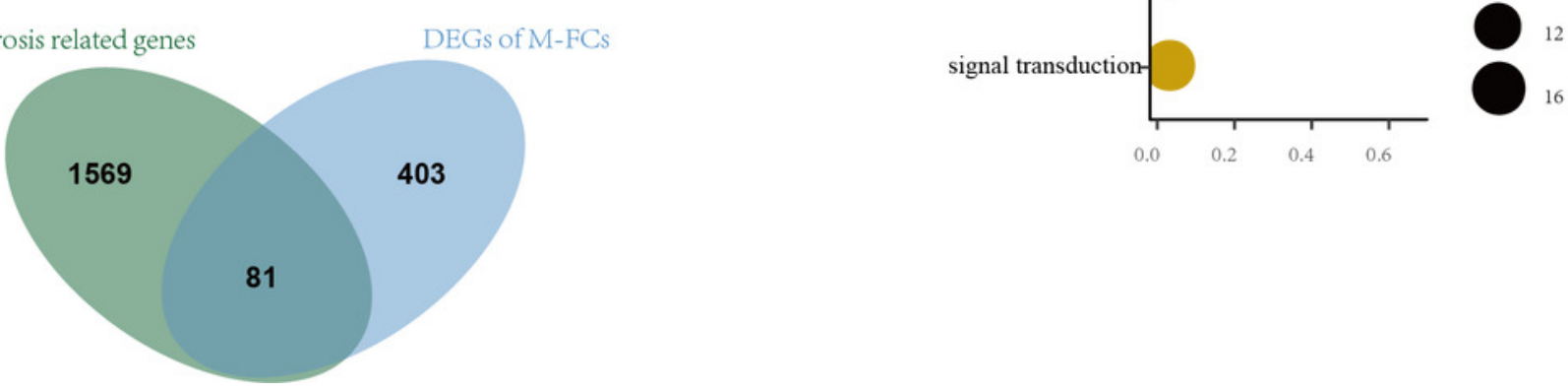


\section{Figure 5}

Identification of co-regulated genes of SMC-FCs and M-FCs.

(A) Venn diagram of common DEGs related to SMC-FCs and M-FCs. (B to E) Cluster

heatmaps of the expression of 15 common genes in the GSE28829, GSE43292, GSE68021, and GSE54666 datasets. Red represents upregulated genes, and green represents downregulated genes. (F) Venn diagram showing the co-downregulated genes and (G) coupregulated genes in the atherosclerosis tissue, SMC-FCs, and M-FCs. SMC-FCs: smooth muscle cell-derived foam cells; M-FCs: macrophage-derived foam cells; and DEGs: differentially expressed genes. 
A

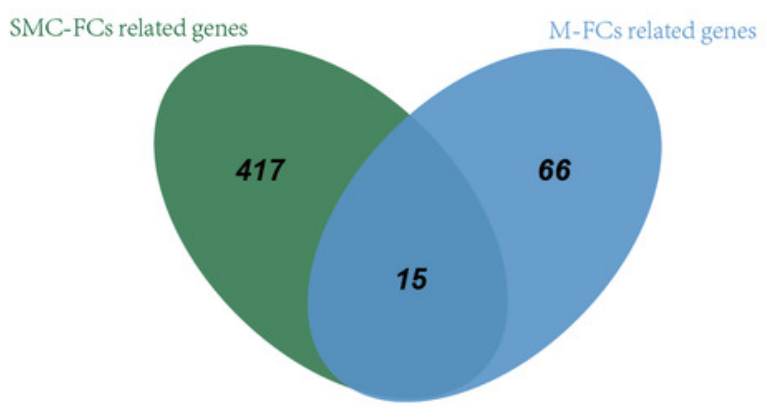

C

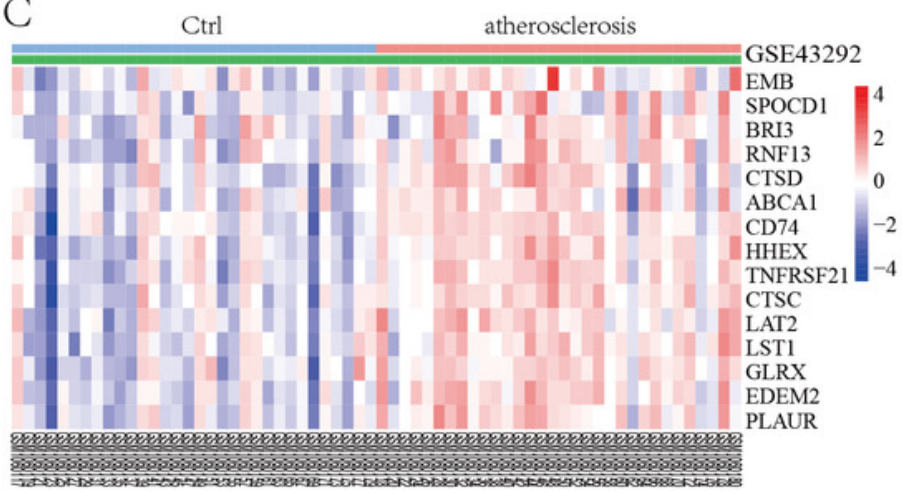

E

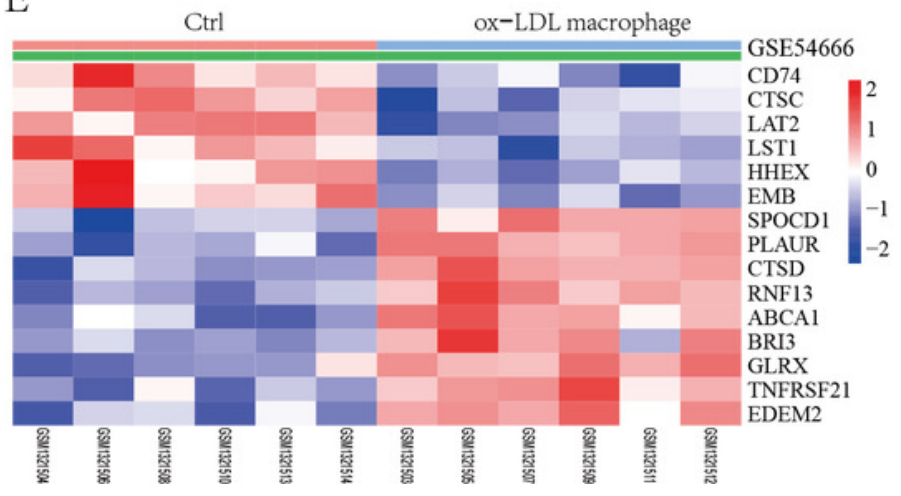

B

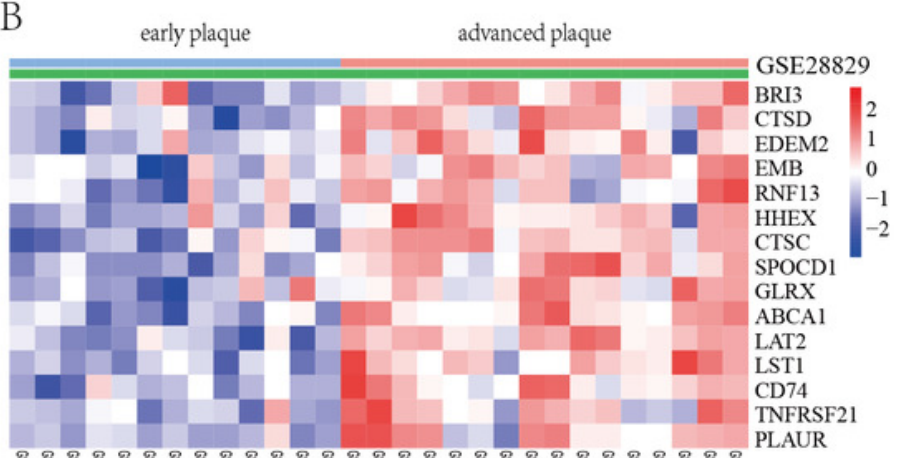

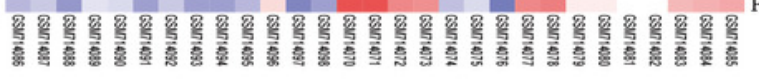

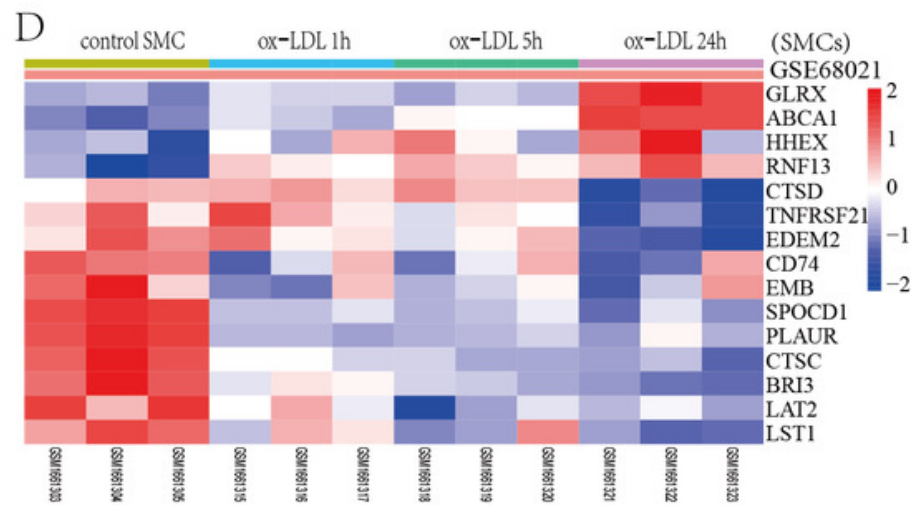

F G

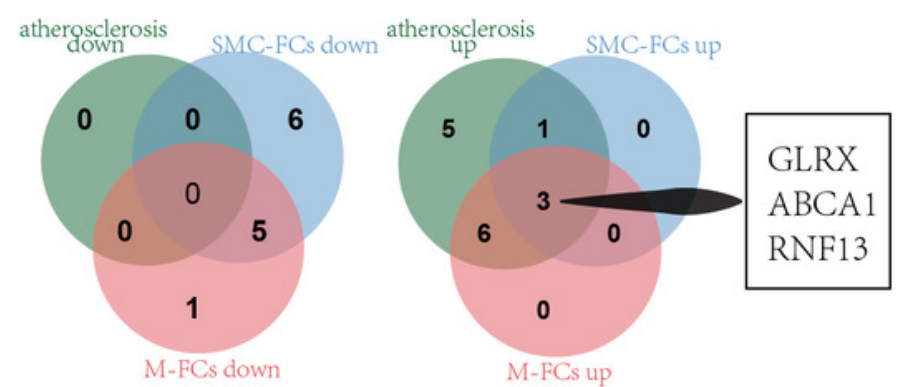




\section{Figure 6}

Validation of the common FCs-related genes and their expression levels in vulnerable atherosclerosis plaques.

Validation of the 3 co-upregulated genes: GLRX, RNF13, and $A B C A 1$, in the macrophages treated with or without ox-LDL (GSE9874) (A). The expression of the 3 genes in unstable plaque samples (B) or ruptured plaque samples $(\mathbf{C})$ compared to stable plaque samples in the GSE120521 or GSE41571 dataset, respectively. ${ }^{*} p<0.05,{ }^{* *} p<0.01,{ }^{* * *} p<0.001$. FC: foam cell.

A

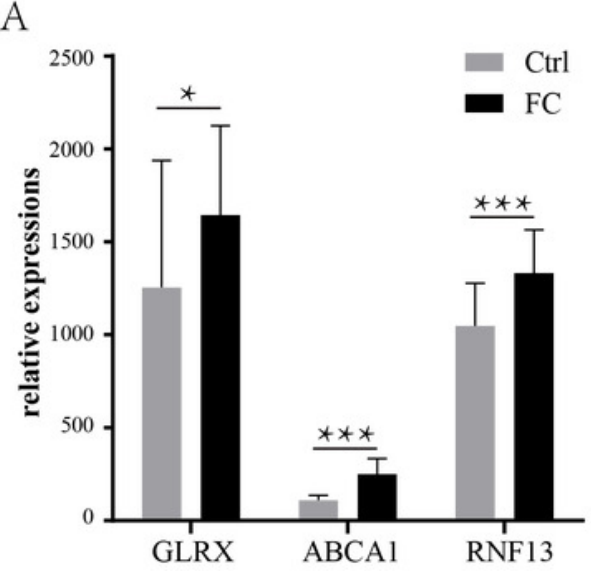

$\mathrm{B}$

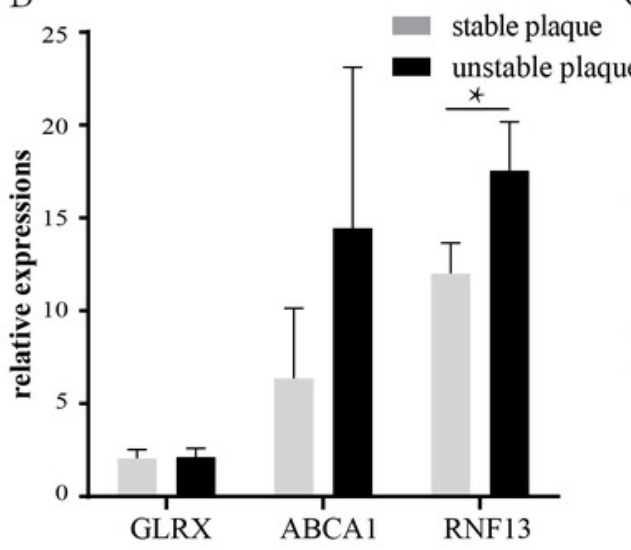

C

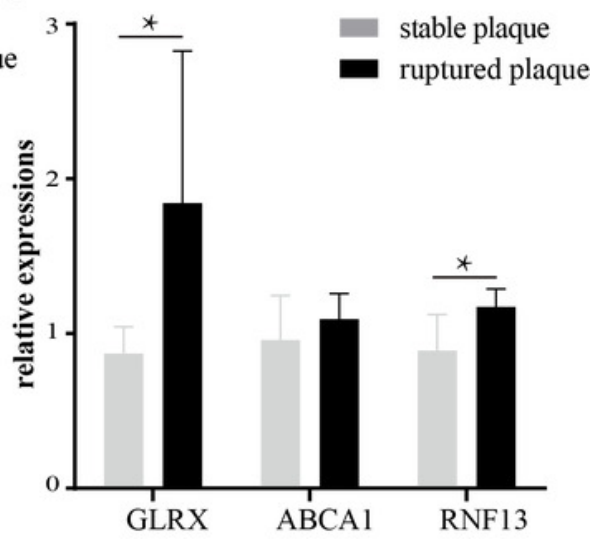

